\title{
Negative priming depends on prime-probe similarity: Evidence for episodic retrieval
}

\author{
ELAINE FOX and JAN WILLEM DE FOCKERT \\ University of Essex, Colchester, England
}

\begin{abstract}
It is well established that requiring a person to respond to a recently ignored object in a visual selection task leads to slower responding (i.e., negative priming). In the present experiment, subjects identified target letters flanked by incompatible distractor letters on prime and probe displays. Prime display distractors appeared as the target letter on one third of subsequent probe displays. We manipulated stimulus strength by means of intensity contrast between letter displays and their background. Displays were presented with either high contrast (white against a black background) or low contrast (dark gray against a black background). The important finding was that negative priming was maximal when prime and probe displays shared the same intensity contrast. These results suggest that greater similarity between prime and probe displays results in improved retrieval of prime display information. The results provide strong support for an episodic retrieval account of negative priming.
\end{abstract}

In the natural environment, biological organisms are frequently confronted with multiple visual objects, only a subset of which are relevant for current behavior. Thus, the ability to select and respond to relevant objects and the ability to successfully ignore irrelevant objects is a fundamental aspect of coherent perceptual-motor processes. Research has shown that perceptual processing of a visual object is greatly facilitated when that object is represented (Treisman, 1992). In other words, attending to an object increases perceptual fluency on subsequent presentations of the same object. This speeding of response to a repeated presentation of an item is often referred to as repetition priming. Two broad classes of explanation can account for repetition priming from attended objects (see Tenpenny, 1995, for review). The most widespread accounts are abstractionist theories, which argue that presentation of an object leads to activation of an abstract mental representation of that object, so that the object's representation becomes more highly accessible (e.g., Morton, 1969). This heightened accessibility leads to faster and more accurate identification of a repeated object relative to a new object. More recently, however, an alternative, but not necessarily contradictory, account has been suggested. Object identification may be driven by retrieval of specific episodic representations (see, e.g., Logan, 1988). On this view, repetition priming is attributed to the retrieval of recent episodes that include the repeated object. Thus, the episodic retrieval approach as-

This research was supported by a grant from the Department of Psychology at the University of Essex. We are grateful to Alastair Neill for help with computer programming and to Ian Smith for help with data collection. We would also like to thank Tram Neill and two anonymous reviewers for helpful comments on the manuscript. Correspondence should be addressed to E. Fox, Department of Psychology, University of Essex, Wivenhoe Park, Colchester CO4 3SQ, England (e-mail: efox@essex.ac.uk). sumes that the brain maintains mental representations of processing episodes rather than abstract representations of objects (see Tenpenny, 1995, for further discussion of abstractionist vs. episodic theories).

Activation of either abstract or episodic representations of objects results in fluent perception and selection of those objects. For many years, theories of visual selective attention assumed that selection of a target from among distracting objects was driven by such excitatory processes. A popular metaphor has been that of a "spotlight" that illuminates or a "zoom lens" that magnifies a particular region of visual space (see, e.g., Eriksen \& Eriksen, 1974). More recently, the intriguing suggestion has been put forward that efficient object identification and selection might be best achieved by means of an excitatory mechanism that is supplemented by an active inhibitory mechanism. The function of this inhibitory mechanism is to suppress distracting information derived from the analysis of irrelevant objects (see, e.g., Neill, 1977; Tipper, 1985). The idea of an active inhibitory mechanism in visual attention was derived largely from the demonstration of negative repetition priming: When an object is ignored in a selection task, subsequent responses to that object are slower and/or less accurate than responses to new objects. Thus, attending to an object seems to facilitate subsequent perception of that object while ignoring an object seems to impair subsequent perception of that object. In a typical negative priming experiment, two displays are presented in rapid succession: The first is generally called the prime, and the second is called the probe. In both displays, the subject attends to one item and attempts to ignore the distracting item. The probe displays of interest are those in which the current target is the same as the prime distractor. Response times (RTs) on these "ignored repetition" (IR) trials are usually slower than those on control (C) trials. Since the seminal studies of Neill (1977) and Tipper (1985), a large amount of research 
has demonstrated that negative priming occurs across a range of stimuli (words, letters, pictures, numbers, nonsense shapes), as well as across a wide variety of task demands (categorization, matching, counting, and target localization; for comprehensive reviews, see Fox, 1995; May, Kane, \& Hasher, 1995; Neill \& Valdes, 1996; Neill, Valdes, \& Terry, 1995; Tipper \& Milliken, 1996). Although the methodological variations across many studies have been valuable in demonstrating the generality of negative priming, this diversity has also created difficulties in drawing comparisons across studies (see, e.g., Fox, 1995; May et al., 1995; Tipper \& Milliken, 1996). This becomes particularly apparent when one considers the mechanisms that can potentially cause negative priming.

As discussed previously, the original inhibition-based explanation of negative priming was essentially an extension of the abstractionist account of repetition priming. The logic was that presentation of a distracting object resulted in activation of an abstract internal representation (on a prime display), and an inhibitory mechanism then decoupled this activated representation from response output. Thus, in contrast to objects that are attended, the abstract mental representations of ignored objects are rendered temporarily inaccessible (see, e.g., Neill, 1977; Tipper, 1985). More recently, a connectionist model of this "inhibitory" mechanism in selective attention has been presented (Houghton \& Tipper, 1994; Houghton, Tipper, Weaver, \& Shore, 1996). A critical feature of inhibitory-based models is that negative priming is seen as occurring in a forward direction from the prime display to the probe display. The mental representation of an ignored object is activated and then suppressed on a prime display, and this suppression carries forward to interfere with identification of the ignored object when it is subsequently presented as a target (e.g., Houghton \& Tipper, 1994). Thus, inhibition-based theories assume that features of the prime display are relatively more important determinants of negative priming than are features of the probe display.

Inhibition-based models assuming activation of attended objects coupled with suppression of irrelevant objects provide a parsimonious account of many selective attentional processes. These models may also provide an important clue regarding the nature of the deficits underlying the individual differences that have been observed in negative priming research. To illustrate, there has been a burgeoning of research that has studied negative priming in clinical and developmental populations. Several studies have shown that older adults (see, e.g., Hasher, Stoltzfus, Zacks, \& Rypma, 1991), children (see, e.g., Tipper, Borque, Anderson, \& Brehaut, 1989), and schizophrenic (see, e.g., Beech, Powell, McWilliam, \& Claridge, 1989) individuals show greater levels of interference in selection tasks and also show less negative priming relative to control groups. Such findings have been influential in the development of theories that particular groups (especially the elderly) are characterized by deficits in inhibitory processes. For example, Hasher and Zacks (1988) have argued that the efficiency of inhibitory processes decreases with increasing age, so the ability to control the contents of "working memory" is lost. The absence of negative priming in older adults is cited as evidence to support this view. However, this conclusion is completely dependent on the assumption that negative priming does indeed reflect the active inhibition of unwanted information and not some other, noninhibitory, process.

In this context, the recent outline of a logical alternative to inhibition-based accounts of negative priming by Tram Neill and his colleagues is of particular importance (Neill \& Valdes, 1992, 1996; Neill, Valdes, Terry, \& Gorfein, 1992). They have extended episodic theories of repetition priming from attended objects (see, e.g., Jacoby, 1983; Logan, 1988) to also account for negative repetition priming from ignored objects. This episodic retrieval account of negative priming is derived from the notion that performance in RT tasks can be mediated by the episodic retrieval of specific "instances" or "episodes" (Logan, 1988). These retrieved instances may contain information about not just the identities of previously processed items but also whether they were responded to or not (Neill \& Valdes, 1992; Neill et al., 1992). The assumption is that negative priming is caused by the automatic retrieval of information from the prime display, which conflicts with the current, correct response (Neill \& Valdes, 1992; Neill et al., 1992). This account emphasizes the role of the probe stimulus as a memory retrieval cue. The idea is that processing the probe target in an ignored repetition condition leads to an automatic retrieval of a representation of a previous processing episode (i.e., the prime display). Episodic representations may contain information about the identity and/or location of objects, their status as "relevant" or "irrelevant," and the responses they require (e.g., "Respond" vs. "Do not respond"). If there is a conflict in the retrieval episode in which an item previously encoded as "irrelevant" on the prime display is now coded as "relevant" on the probe display, slower RTs occur because of the mismatch between processing episodes and not because of inhibition applied during the prime display. Neill et al. (1992) also pointed out that responses on ignored repetition trials may be slowed because the lack of correct response information may force subjects to rely on slower "algorithmic" processing to elicit the appropriate response (cf. Logan, 1988). A critical feature of this account is that negative priming is considered to be the result of a backward operating effect, with the probe target acting as a memory cue. Importantly, the episodic retrieval account can explain negative repetition priming effects without the need for any inhibitory mechanism in selective attention. The importance of this account of negative priming is that it raises the possibility that the individual differences noted above may be due to deficits in episodic retrieval rather than to deficits in an inhibitory mechanism. Thus, a crit- 
ical question concerns whether negative (and positive) repetition priming effects are caused by activation (or suppression) of abstract representations or whether they are produced by the retrieval of episodic representations of specific processing episodes.

The strongest evidence for an episodic retrieval account of negative priming comes from delay effects (Neill et al., 1992) and long-term negative priming from novel shapes (DeSchepper \& Treisman, 1996). Neill et al. (1992) examined the effect of delay prior to the prime display (i.e., the temporal delay, 500 or $4,000 \mathrm{msec}$, between the prime display and the immediately preceding display) in addition to delay effects after the prime display $(500$ or $4,000 \mathrm{msec}$ ). There was no difference in the level of negative priming between the 500/500 and the 4,000/4,000 conditions (i.e., no decay of negative priming). Negative priming was strongest when the prime display was preceded by a long interval and followed by a short interval $(4,000 / 500)$ and weakest when the prime display was preceded by a short interval and followed by a long interval $(500 / 4,000)$. This pattern of results indicates that the negative priming effect depended on the temporal discriminability of the prime display. This dependence of negative priming on the delay prior to a prime display is difficult to account for from an inhibition-based perspective but follows naturally from an episodic retrieval approach (Neill et al., 1992). It should be noted that there is one published failure to replicate these results (Hasher, Zacks, Stoltzfus, Kane, \& Connelly, 1996). However, in the Hasher et al. study, delay intervals of 500 and $2,500 \mathrm{msec}$ were used rather than 500 and $4,000 \mathrm{msec}$. It is possible that the 2,500 delay may not have been as salient as the 4,000 -msec delay used by Neill et al. (1992), resulting in a failure to replicate. Additional support for an episodic retrieval account comes from the recent demonstration of longlasting negative priming effects after a single trial in which a novel shape was ignored (DeSchepper \& Treisman, 1996). Subjects were required to match a green nonsense shape on the left with a white shape on the right. The green shape was spatially overlapped with a distracting red shape that subjects were instructed to ignore. However, if the ignored red shape was subsequently presented as an attended green shape 1 trial later, 100 trials later, 200 trials later, and even 1 month later, significant negative priming occurred. The critical finding was that the magnitude of negative priming did not decay significantly over hundreds of trials (DeSchepper \& Treisman, 1996). Once again, it is difficult to see how selective inhibition can account for negative priming effects that last up to 1 month. Retrieval of an episodic trace that contains some memory of whether the novel item was previously attended or ignored seems the most logical explanation.

Another possible avenue to investigate episodic retrieval accounts of negative priming is to manipulate prime-probe similarity. It is generally accepted that episodic retrieval is determined by the similarity of context between encoding and retrieval episodes (Tulving, 1983). In a negative priming task, the similarity of context between the prime display (encoding) and the probe display (retrieval) can be manipulated. If episodic retrieval is a primary determinant of negative priming, then priming should occur to the degree that there is similarity of context on prime and probe displays. Evidence for this has been presented in a recent study reported by Neill (1997). Subjects were required to identify a centrally located target letter while ignoring distracting letters on the left and the right of the target. The distracting letters were presented either simultaneously with the target (early) or $400 \mathrm{msec}$ later (late) on both prime and probe displays. As predicted by the episodic retrieval account, negative priming occurred only when the distractor onset matched across prime and probe displays (early/early, late/late). The logic of the present experiment is similar to that of Neill (1997), using a different manipulation of context. We wanted to test the hypothesis that a probe display will be a more effective retrieval cue when it is highly similar to the previous prime display, thus leading to more negative priming. Contextual similarity was manipulated by means of varying the stimulus intensity (or contrast) between target and distractor stimuli and the background. Thus, white stimuli against a black background provided high contrast, while dark gray stimuli against a black background provided low contrast. Contrast was manipulated on both prime and probe displays, leading to four experimental conditions: high-contrast prime followed by high-contrast probe (high/high), high-contrast prime followed by low-contrast probe (high/low), low-contrast prime followed by high-contrast probe (low/high), and low-contrast prime followed by low-contrast probe (low/ low). While both selective inhibition and episodic retrieval accounts predict greater negative priming with high-contrast prime displays, only the episodic retrieval account predicts greater negative priming when the prime and probe displays are similar (i.e., high/high and low/ low) than when they are dissimilar (i.e., high/low and low/high).

\section{METHOD}

\section{Subjects}

Twenty-four undergraduate students at the University of Essex participated in a single experimental session of about $30 \mathrm{~min}$ in return for $£ 2$. All were between 18 and 28 years of age and had normal or correctedto-normal vision.

\section{Apparatus and Stimuli}

Stimulus presentation and data collection were controlled by a Macintosh LCIII computer. The $D, F, J$, and $K$ keys of the computer keyboard were used to register subjects' responses, and response latencies and errors were measured by means of the SuperLab computer program. Target and distractor stimuli were the capital letters $S, X, O$, or $I$, each subtending about $.92^{\circ}$ of vertical and $.57^{\circ}$ of horizontal visual angle. Letters were always presented in triplets with the target letter in the middle, flanked on either side by the same distractor letter (e.g., SXS). The center-to-center distance from the target letter to the flanking letter on each side was $1.5^{\circ}$ of visual angle at a viewing distance of approxi- 
mately $50 \mathrm{~cm}$. The background screen was always black. In the highcontrast displays, target and distractor letters were presented in white against the black background. In low-contrast displays, target and distractor letters were presented in dark gray against the black background.

\section{Procedure}

Each subject participated in a 30-min session consisting of five blocks of 72 trials each with the first trial block being considered as practice. Subjects were instructed to identity the middle letter of each letter triplet by pressing the appropriate key on the computer keyboard $(D, F, J$, and $K$ for the letters $S, X, O$, and $I$, respectively). Instructions emphasized accuracy as well as speed. Each trial consisted of a prime and a probe display, each of which contained a letter triplet. The sequence of events within a trial was as follows: A fixation display $(+++)$ was presented at the center of the screen for $500 \mathrm{msec}$. This display was replaced by the prime display for $150 \mathrm{msec}$. This was followed by a blank screen until a response was made or until $2,000 \mathrm{msec}$ had elapsed. Following the response (or after $2,000 \mathrm{msec}$ ), there was a blank screen for $350 \mathrm{msec}$, and then the probe display was presented in the same location for $150 \mathrm{msec}$. A blank screen was then presented until a response was made or until $2,000 \mathrm{msec}$ had elapsed. There was an intertrial interval of $1,000 \mathrm{msec}$.

\begin{abstract}
Design
The experimental design included the within-subjects factors of prime contrast (high vs. low), probe contrast (high vs. low), and trial type. The two critical trial types were as follows: (1) control (C), in which all the stimuli on prime and probe displays were unrelated (e.g. $S X S$ followed by $O I O$ ), and (2) ignored repetition (IR), in which the distractor on the prime display was repeated as the target on the probe display (e.g., SXS followed by $O S O$ ). In each block, $33 \%$ of trials were IR and $67 \%$ were C. All of the prime and probe displays contained a distractor that was different from the target (e.g., XSX).
\end{abstract}

\section{RESULTS}

\section{Prime Displays}

Median RTs for correct responses were calculated in each condition for each subject. RTs on high-contrast prime displays were faster $(586 \mathrm{msec})$ than were those on low-contrast prime displays $(607 \mathrm{msec})[t(23)=-4.6$, $S E M=4.537, p<.001]$. Error rates were in the same direction as the RTs ( 1.9 vs. 2.5 , respectively), although these were not significantly different $[t(23)=1.81$, SEM $=$ $.0033, p<.09]$. Thus, there was no evidence of any speedaccuracy tradeoff.

\section{Probe Displays}

Median RTs for correct responses as a function of prime contrast, probe contrast, and trial type are presented in Table 1. These median RTs were entered into a 2 (prime contrast: high vs. low) $\times 2$ (probe contrast: high vs. low) $\times 2$ (trial type: $C$ vs. IR) analysis of variance (ANOVA) with subjects as a random factor. RTs on high-contrast probe displays were faster $(608 \mathrm{msec})$ than were those on low-contrast probe displays $(647 \mathrm{msec})[F(1,23)=$ $\left.59.6, M S_{\mathrm{e}}=1,186.5, p<.001\right]$. There was also a significant main effect for trial type, with slower RTs on IR $(635 \mathrm{msec})$ relative to those on $\mathrm{C}(620 \mathrm{msec})$ trials $\left[F(1,23)=9.9, M S_{\mathrm{e}}=998.6, p<.01\right]$. There were significant two-way interactions between prime contrast and probe contrast $\left[F(1,23)=4.3, M S_{\mathrm{e}}=708.1, p<.05\right]$ and between prime contrast and trial type $[F(1,23)=$ $7.7, M S_{\mathrm{e}}=230.8, p<.01$; more negative priming following high-contrast primes relative to low-contrast primes, with 20 and $8 \mathrm{msec}$, respectively]. However, as predicted by the episodic retrieval hypothesis, these interactions were qualified by a significant prime contrast $\times$ probe contrast $\times$ trial type interaction $\left[F(1,23)=9.8, M S_{\mathrm{e}}=\right.$ $540.2, p<.01]$. This interaction is illustrated in Figure 1. Planned comparisons confirmed the pattern of results shown in Figure 1. Significant levels of negative priming (longer RTs on IR relative to C trials) occurred for highcontrast probes following high-contrast primes $[t(23)=$ $-3.77, S E M=8.05, p<.001]$ and for low-contrast probes following low-contrast primes $[t(23)=-3.16, S E M=$ $6.15, p<.004]$. The magnitude of negative priming in the two mismatch conditions did not reach statistical significance.

The mean error percentages for each condition are also presented in Table 1. The arcsin transformations of these errors were subjected to the same prime contrast $\times$ probe contrast $\times$ trial type ANOVA as the RT data. There was a main effect for prime contrast $\left[F(1,23)=4.9, M S_{\mathrm{e}}=\right.$ $.007, p<.05]$ such that errors on the probe display were more frequent following high-contrast prime displays (5.2) than following low-contrast probe displays (4.7). There was a trend for more errors on IR (5.3) relative to $C(4.5)$ trials $\left[F(1,23)=3.4, M S_{\mathrm{e}}=.025, p<.08\right]$, and there was a significant prime contrast $\times$ trial type interaction $\left[F(1,23)=4.5, M S_{\mathrm{e}}=.006, p<.05\right]$. As in the RT analysis, this interaction was due to more negative priming occurring on probe displays following high-contrast prime displays (2.0) relative to those following lowcontrast prime displays $(0.0)$. The critical three-way interaction among prime contrast, probe contrast, and trial type was not significant $\left[F(1,23)=2.5, M S_{\mathrm{e}}=.014, p<.12\right]$. There was no evidence of any speed-accuracy tradeoffs.

\section{DISCUSSION}

The present results provide strong support for the episodic retrieval account of negative priming. Significant negative priming occurred only

Table 1

Means and Standard Deviations of Median Correct Probe Reaction Times (RT, in Milliseconds) and Percentage of Errors (PE) as a Function of Prime Contrast, Probe Contrast, and Trial Type

\begin{tabular}{|c|c|c|c|c|c|c|c|c|c|c|c|c|c|c|c|c|}
\hline \multirow{3}{*}{ Trial Type } & \multicolumn{16}{|c|}{ Prime-Probe Contrast } \\
\hline & \multicolumn{4}{|c|}{$\mathrm{High} / \mathrm{High}$} & \multicolumn{4}{|c|}{ High/Low } & \multicolumn{4}{|c|}{ Low/High } & \multicolumn{4}{|c|}{ Low/Low } \\
\hline & \multicolumn{2}{|c|}{ RT } & \multicolumn{2}{|c|}{ PE } & \multicolumn{2}{|c|}{ RT } & \multicolumn{2}{|c|}{$\mathrm{PE}$} & \multicolumn{2}{|c|}{ RT } & \multicolumn{2}{|c|}{$\mathrm{PE}$} & \multicolumn{2}{|c|}{ RT } & \multicolumn{2}{|c|}{ PE } \\
\hline Control & 600 & 69.3 & 4.0 & 0.32 & 640 & 61.4 & 5.0 & 0.55 & 603 & 72.7 & 4.5 & 0.45 & 638 & 64.5 & 4.7 & 0.45 \\
\hline Ignored repetition & 630 & 64.3 & 6.4 & 0.55 & 650 & 57.7 & 5.5 & 0.55 & 600 & 77.5 & 4.0 & 0.32 & 658 & 56.4 & 5.5 & 0.55 \\
\hline
\end{tabular}




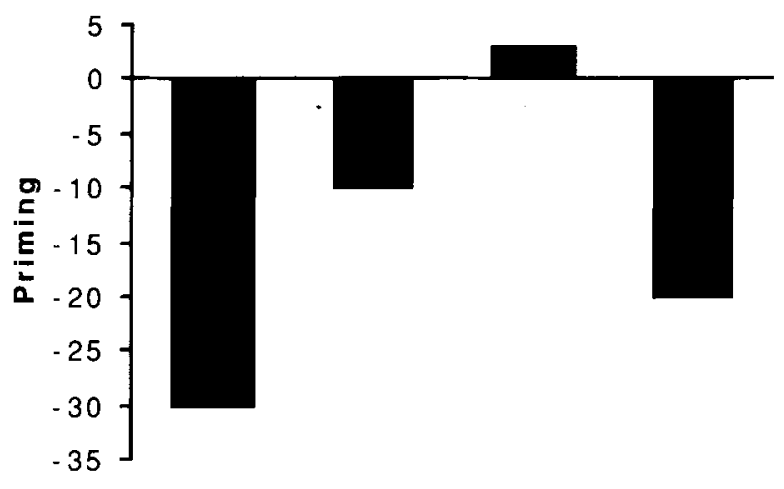

High/ High High/Low Low/ High Low/Low

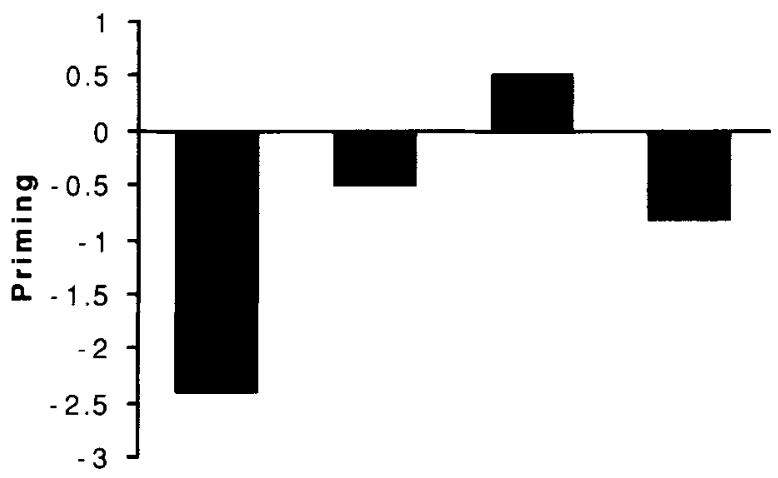

High/ High High/Low Low/ High Low/Low

Prime/Probe Contrast

Figure 1. Negative priming (i.e., the difference between control and ignored repetition trials) for reaction time (upper panel) and errors (lower panel) as a function of prime-probe contrast.

when the prime and probe displays were similar in terms of contrast. When the probe display was high contrast, negative priming occurred when the prime display had also been high contrast $(-30 \mathrm{msec})$, but not when the prime display had been low contrast $(+3 \mathrm{msec})$. However, when the probe display was low contrast, significant negative priming occurred only when the prime display had also been low contrast $(-20 \mathrm{msec})$ and not when the prime display had been high contrast $(-10 \mathrm{msec})$. Thus, although highcontrast prime distractors did produce more negative priming overall than low-contrast prime distractors, as predicted by both episodic retrieval and inhibition-based accounts, the dependence of negative priming on primeprobe similarity is best explained by the episodic retrieval account. We should point out that we are not claiming that negative priming would never occur when prime and probe displays are not closely matched; more statistical power might very well yield an effect in such conditions. The important point is that negative priming is maximal when prime and probe displays are matched in terms of contrast and minimal when there is little contextual match between them.

In the present study, stimulus contrast itself had a significant impact on RTs, with high-contrast displays being responded to faster than lowcontrast displays. It has been pointed out that systematic variations in RTs to prime displays across different conditions makes it difficult to draw firm conclusions concerning negative priming effects in different probe conditions (Tipper \& Milliken, 1996). The contrast manipulation makes such variations inevitable, however, as targets in high-contrast trials are easier to select than are targets in low-contrast trials. Importantly, however, the three-way interaction between prime contrast, probe contrast, and priming demonstrates that negative priming occurred following both high-contrast primes (high/high) and low-contrast primes (low/low). The critical determinant of negative priming was the contextual similarity of prime and probe displays in this experiment and not just the nature of processing on the prime display (see also Neill, 1997).
As noted previously, a greater magnitude of negative priming from high-contrast prime displays can be accounted for by either inhibitionbased or episodic retrieval approaches. According to an episodic retrieval account, negative priming occurs because access to an episodic representation of a prime display that contains a "do-not-respond" tag interferes with the existing "respond" tag. Greater accessibility of the episodic representation will lead to easier retrieval and hence larger negative priming effects. Because bright stimuli are likely to be processed more extensively than are dim stimuli (see Houghton et al., 1996), the episodic retrieval account predicts more negative priming from high-contrast prime displays than from low-contrast prime displays. Inhibition-based accounts can also explain why greater negative priming occurs from high-contrast relative to low-contrast prime displays. For example, the Houghton and Tipper model (Houghton \& Tipper, 1994; Houghton et al., 1996) explicitly states that strong distractors will produce stronger interference than weak distractors and are therefore expected to be more strongly inhibited, producing increased negative priming. Thus, high-contrast (strong) distractors would be expected to produce more negative priming than low-contrast (weak) distractors (Houghton et al., 1996; present experiment). ${ }^{1}$ The critical result that separates the two accounts is the dependence of negative priming on the contextual similarity between the prime and probe displays. It is especially striking that significant negative priming occurred in the low/low condition but not in the high/low condition. Thus, while the "strength" of prime display distractors can have a strong effect on negative priming, these effects can be overridden by powerful cues at retrieval (i.e., low-contrast prime distractors can produce more negative priming than high-contrast prime distractors when the probe display is also low contrast). This result suggests that the negative priming in this experiment was driven by the retrieval of episodic representations containing response information, as proposed by Neill et al. (1992).

The finding that retrieval processes can play a role in producing negative priming effects is important because the absence of negative priming in older adults is widely cited as evidence for deficits in the ability to selectively inhibit distracting information in this population. Similar explanations have been put forward for findings that other clinical populations (e.g., schizophrenia) show reduced negative priming effects. It may well be that these subgroups are indeed characterized by deficits in an inhibitory component of selective attention. However, the ambiguity of what mechanism causes the negative priming effect suggests some caution in drawing such a conclusion. The present results add further weight to the alternative hypothesis that older adults may have deficits in their ability to retrieve episodic traces rather than deficits in selective inhibition.

As a result of its backward acting nature, negative priming produced by episodic retrieval depends largely on two factors: (1) the extent to which the probe display serves as a retrieval cue for the previous prime display and (2) whether the prime display is easily discriminable in episodic memory. That the temporal discriminibility of the prime affects negative priming is suggested by the dependence of negative priming on the interval prior to the prime display (Neill et al., 1992; but see Hasher et al, 1996). The extent to which the probe serves as a retrieval cue depends on the similarity between the prime (encoding) and the probe (retrieval) displays. The recent experiment reported by Neill (1997) demonstrated that negative priming depended on the contextual similarity of early presence or absence of distractors on prime and probe displays. Moreover, Neill (1997) also found that prime-probe similarity had equivalent effects on repetition priming from attended letters, suggesting that a similar episodic retrieval mechanism underlies repetition and negative priming. The present experiment provides further support for an episodic retrieval account of negative priming. Negative priming was reliable only when the contrast (brightness) of a display matched across prime and probe displays. Thus, probe displays that are similar in terms of contrast to prime displays provide a better retrieval cue than do dissimilar probe displays and therefore produce greater levels of negative priming. We have recently heard of unpublished data from Stoltz and Neely, who also manipulated stimulus intensity across prime and probe displays in a letter-matching task. As in the present experiment, they found that negative priming occurred only when the stimulus intensities of prime and probe trials matched (Neely, personal communication, December 1996).

Thus, it seems that there is mounting evidence that more negative priming occurs when prime and probe displays have a similar context 
than when the context differs. ${ }^{2}$ This effect is in line with the episodic retrieval account of negative priming, which suggests that a probe context that is similar to the prime context provides a better retrieval cue than a probe context that differs from the prime context. In IR trials, this facilitated retrieval causes an enhanced negative priming effect, consistent with the present findings. Episodic retrieval seems the best explanation of the present data. However, Houghton et al. (1996) pointed out that their inhibition-based model predicts that increased similarity between prime distractors and probe targets should result in increased negative priming because the various attributes of a distractor, such as its location or shape, are inhibited separately. A probe target that shares a large number of characteristics with a prime distractor will produce strong negative priming. Although inhibition might indeed spread to various attributes of a distracting stimulus, it seems unlikely that inhibition would also spread to the surrounding context. Therefore, it is difficult to account for the present results, showing that contextual similarity between prime and probe displays is a critical determinant of negative priming, with inhibition-based models. Such effects are, however, predicted by episodic retrieval models. Thus, in the present experiment at least, it seems that negative priming was driven primarily by retrieval of episodic traces of previous prime episodes or instances.

It is of course possible that both episodic retrieval and selective inhibition underlie negative priming effects (see, e.g., May et al., 1995 Milliken, Tipper, \& Weaver, 1994). However, the present results would seem to eliminate a strong inhibition-based account of negative priming. For example, the dependence of negative priming on contextual similarity across prime and probe display is difficult to reconcile with the notion that abstract representations of distracting objects are actively suppressed at Time 1 (prime) and that this inhibition carries forward to interfere with responses at Time 2 (probe). The fact that randomly presented changes on the probe display (e.g., high or low contrast) can change the magnitude of negative priming is not compatible with a forward-acting inhibition of abstract representations. A strong version of inhibition-based negative priming assuming abstract representations would predict no effect of physical similarity or contex on negative priming. These factors, however, clearly do influence the magnitude of negative priming observed (Neill, 1997; present experiment). A weaker version of inhibition-based negative priming that does not assume that abstract representations are inhibited might be able to explain the present results. It is possible that the internal representations of distracting objects are inhibited at Time 1 but that the representations are highly specific (i.e., less abstract) in that they may contain detailed information on specific features of the ignored object. It could then be the case that inhibition would be stronger when there is a greater match across prime and probe displays. Thus, while we accept that the present results do not disconfirm an inhibition-based account of negative priming, we believe that the present results do implicate memory retrieval as playing a critical role in negative priming (see also Milliken et al. 1994). It may well be the case that inhibitory processes are most important during the initial selection of a target object, whereas retrieval processes are more important in sequential responding. What gets retrieved on the probe trial may indeed be the memory of previous inhibition. It is an important task for future research to attempt to distinguish between inhibition and episodic retrieval mechanisms and perhaps to determine how the two mechanisms may interact in coordinating perceptual-motor interactions with the environment. As things stand at the moment, however, it seems that an epdisodic retrieval account can explain most of the extant data in the negative priming literature and is supported in particular by the effects of delays prior to a prime display (Neill et al., 1992), long-term negative priming from novel shapes (DeSchepper \& Treisman, 1996), and prime-probe contextual similarity (Neill, 1997; present experiment).

\section{REFERENCES}

Beech, A., Powell, T., McWilliam, J., \& Claridge, G. (1989). Evidence of reduced "cognitive inhibition" in schizophrenia. British Journal of Clinical Psychology, 28, 109-116.

DeSchepper, B., \& Treisman, A. (1996). Visual memory for novel shapes: Implicit coding without attention. Journal of Experimental Psychology: Learning, Memory, \& Cognition, 22, 27-47.

Eriksen, B. A., \& Eriksen, C. W. (1974). Effects of noise letters upon the identification of a target letter in a nonsearch task. Perception \& Psychophysics, 16, 143-149.

Fox, E. (1995). Negative priming from ignored distractors in visual selection: A review. Psychonomic Bulletin \& Review, 2, 145-173.

Hasher, L., Stoltzfus, E. R., Zacks, R. T., \& Rypma, B. (1991). Age and inhibition. Journal of Experimental Psychology: Learning, Memory, \& Cognition, 17, 163-169.

HASHER, L., \& ZACKS, R. T. (1988). Working memory, comprehension, and aging: A review and a new view. In G. H. Bower (Ed)., The psychology of learning and motivation (Vol. 22, pp. 193-225). New York: Academic Press.

Hasher, L., Zacks, R. T., Stoltzfus, E. R., Kane, M. J., \& Connelly, S. L. (1996). On the time course of negative priming: Another look. Psychonomic Bulletin \& Review, 3, 231-237.

Houghton, G., \& TIPPER, S. P. (1994). A model of inhibitory mechanisms in selective attention. In D. Dagenbach \& T. Carr (Eds.), Inhibitory mechanisms in attention, memory and language (pp. 53-112). San Diego: Academic Press.

Hovghton, G., Tipper, S. P., Weaver, B., \& SHore, D. I. (1996). Inhibition and interference in selective attention: Some tests of a neural network model. Visual Cognition, 3, 119-164.

JACOBY, L. L. (1983). Perceptual enhancement: Persistent effects of an experience. Journal of Experimental Psychology: Learning, Memorv, \& Cognition, 9, 21-38.

LoGAN, G. D. (1988). Toward an instance theory of automatization Psychological Review, 95, 492-527.

May, C. P., Kane, M. J., \& Hasher, L. (1995). Determinants of negative priming. Psychological Bulletin, 118, 35-54.

Milíiken, B., TIPPER, S. P., \& WeAver, B. (1994). Negative priming in a spatial localization task: Feature mismatching and distractor inhibition. Journal of Experimental Psychology: Human Perception \& Performance, 20, 624-646.

MORTON, J. (1969). Interaction of information in word recognition. Psychological Review, 76, 165-178.

NEILL, W. T. (1977). Inhibitory and facilitatory processes in selective attention. Journal of Experimental Psychology: Human Perception \& Performance, 3, 444-450.

NEILL, W. T. (1997). Episodic retrieval in negative priming and repetition priming. Journal of Experimental Psychology: Learning, Memory, \& Cognition, 23, 1291-1305.

NeILl, W. T., \& VALDES, L. A. (1992). Persistence of negative priming Steady state or decay? Journal of Experimental Psychology: Learning, Memory, \& Cognition, 18, 565-576.

Neill, W. T., \& VAldes, L. A. (1996). Facilitatory and inhibitory aspects of attention. In A. F. Kramer, M. G. H. Coles, \& G. D. Logan (Eds.), Converging operations in the study of visual selective attention (pp. 77-106). Washington, DC: American Psychological Association.

Neill, W. T., Valdes, L. A., \& Terry, K. M. (1995). Selective attention and the inhibitory control of cognition. In F. N. Dempster \& C. J. Brainerd (Eds.), New perspectives on interference and inhibition in cognition (pp. 207-261). New York: Academic Press.

Neill, W. T., Valdes, L. A., Terry, K. M., \& Gorfein, D. S. (1992). Persistence of negative priming: II. Evidence for episodic trace retrieval. Journal of Experimental Psychology: Learning, Memory, \& Cognition, 18, 993-1000.

TENPENNY, P. L. (1995). Abstractionist versus episodic theories of repetition priming and word identification. Psvchonomic Bulletin \& Review, 2, 339-363

TIPPER, S. P. (1985). The negative priming effect: Inhibitory priming by ignored objects. Quarterly Journal of Experimental Psychology, 37A, 571.590 .

Tipper, S. P., Borque, T. A., Anderson, S. H., \& Brehaut, J. C. (1989). Mechanisms of attention: A developmental study. Journal of Experimental Child Psychology, 48, 353-378

TIPPER, S. P., \& MillikeN, B. (1996). Distinguishing between inhibitionbased and episodic retrieval-based accounts of negative priming. In 
A. F. Kramer, M. G. H. Coles, \& G. D. Logan (Eds.), Converging operations in the study of visual selective attention (pp. 337-363) Washington, DC: American Psychological Association.

Treisman, A. M. (1992). Perceiving and re-perceiving objects. American Psvchologist, 47, 862-875.

Tulving, E. (1983). Elements of episodic memory. Oxford: Oxford University Press.

\section{NOTES}

1. It should be noted that it is not entirely clear what the Houghton and Tipper model (Houghton \& Tipper, 1994; Houghton et al., 1996) would predict with regard to the effect of stimulus intensity of prime distractors. One prediction is that stronger distractors (i.e., high contrast) will produce more negative priming. However, the model also predicts that when the prime target is difficult to select, more negative priming should occur. Since low-contrast prime displays result in more difficult selection (longer RTs and more errors), it might be expected that distractors should be more strongly inhibited under these conditions. Therefore, it is not clear what the Houghton and Tipper model would predict for the present experiment.

2. We should point out here that we have tried to replicate the present results by manipulating display color (red or blue) across prime and probe displays instead of stimulus intensity. We were unable to replicate the present results. However, we also found no negative priming at all in the color experiment, which implies that the subjects in that experiment may not have been typical. We are confident that the present results with contrast are replicable, especially since Stolz and Neely found a similar pattern.

(Manuscript received December 23, 1996; revision accepted for publication May 14, 1997.) 